\title{
On the Stanley-Wilf conjecture for the number of permutations avoiding a given pattern
}

\author{
Richard Arratia \\ Department of Mathematics \\ University of Southern California \\ Los Angeles, CA 90089-1113 \\ email: rarratia@math.usc.edu
}

Submitted: July 27, 1999; Accepted: August 25, 1999.

\begin{abstract}
Consider, for a permutation $\sigma \in \mathcal{S}_{k}$, the number $F(n, \sigma)$ of permutations in $\mathcal{S}_{n}$ which avoid $\sigma$ as a subpattern. The conjecture of Stanley and Wilf is that for every $\sigma$ there is a constant $c(\sigma)<\infty$ such that for all $n, F(n, \sigma) \leq c(\sigma)^{n}$. All the recent work on this problem also mentions the "stronger conjecture" that for every $\sigma$, the limit of $F(n, \sigma)^{1 / n}$ exists and is finite. In this short note we prove that the two versions of the conjecture are equivalent, with a simple argument involving subadditivity.

We also discuss $n$-permutations, containing all $\sigma \in \mathcal{S}_{k}$ as subpatterns. We prove that this can be achieved with $n=k^{2}$, we conjecture that asymptotically $n \sim(k / e)^{2}$ is the best achievable, and we present Noga Alon's conjecture that $n \sim(k / 2)^{2}$ is the threshold for random permutations.
\end{abstract}

Mathematics Subject Classification: 05A05,05A16.

\section{INTRODUCTION}

Consider, for a permutation $\sigma \in \mathcal{S}_{k}$, the set $\mathcal{A}(n, \sigma)$ of permutations $\tau \in \mathcal{S}_{n}$ which avoid $\sigma$ as a subpattern, and its cardinality, $F(n, \sigma):=|\mathcal{A}(n, \sigma)|$. Recall that " $\tau$ contains $\sigma$ " as a subpattern means that there exist $1 \leq x_{1}<x_{2}<\cdots<x_{k} \leq n$ such that for $1 \leq i, j \leq k$,

$$
\tau\left(x_{i}\right)<\tau\left(x_{j}\right) \text { if and only if } \sigma(i)<\sigma(j) .
$$

An outstanding conjecture is that for every $\sigma$ there is a finite constant $c(\sigma)$ such that for all $n, F(n, \sigma) \leq c(\sigma)^{n}$. In the 1997 Ph.D. thesis of Bóna [2], supervised by

The author thanks Noga Alon, Béla Bollobás, and Miklós Bóna for discussions of this problem. 
Stanley, this conjecture is attributed to "Wilf and Stanley [oral communication] from 1990." All the recent work on this problem also mentions the "stronger conjecture" that for every $\sigma$, the limit of $F(n, \sigma)^{1 / n}$ exists and is finite. According to Wilf (private communication, 1999) the original conjecture was of this latter form.

In this short note we give, as Theorem 1, a simple argument, involving subadditivity, which shows that the two versions of the conjecture are equivalent.

Here is some background information on the current status of the Stanley-Wilf conjecture. Represent $\sigma \in \mathcal{S}_{k}$ by the word $\sigma(1) \sigma(2) \cdots \sigma(k)$. For the case of the increasing pattern, i.e the identity permutation, $\sigma=12 \cdots k$, the upper bound $F(n, \sigma) \leq\left((k-1)^{2}\right)^{n}$ is well known, and follows from the Robinson-Schensted-Knuth correspondence; also Regev [7] gives the asymptotics

$$
F(n, 12 \cdots k) \sim \lambda_{k} \frac{(k-1)^{2 n}}{n^{k(k-2) / 2}}
$$

with an explicit constant $\lambda_{k}$. Simion and Schmidt [8] give a bijective proof that for each $\sigma \in \mathcal{S}_{3}, F(n, \sigma)=\frac{1}{n+1}\left(\begin{array}{c}2 n \\ n\end{array}\right)$; see also Knuth [6], section 2.2.1, exercises.

For $\sigma=1342$, Bóna [2] finds the explicit generating function for $F(n, \sigma)$, showing that for all $n, F(n, 1342)<8^{n}$, and $\lim F(n, 1342)^{1 / n}=8$. Note in contrast that $\lim F(n, 1234)^{1 / n}=9$. Bóna observes that indeed, in all cases for which $\lim F(n, \sigma)^{1 / n}$ is known explicitly, it is an integer! For the special class of "layered patterns," such as $\sigma=6734512$, Bóna [3] has shown that $\sup _{n} F(n, \sigma)^{1 / n}$ is finite. Alon and Friedgut [1] prove an upper bound for the general case which is tantalizingly close to the goal; they relate the problem to a result on generalized Davenport-Schinzel sequences from Klazar [5], and show that for every $\sigma \in \mathcal{S}_{k}$ there exists $c(\sigma)<\infty$ such that for all $n, F(n, \sigma) \leq c(\sigma)^{n \gamma^{*}(n)}$, where $\gamma^{*}(n)$ is an extremely slowly growing function, given explicitly in terms of the inverse of the Ackermann function.

Theorem 1. For every $k \geq 2$ and $\sigma \in \mathcal{S}_{k}$, for every $m, n \geq 1$,

$$
F(m+n, \sigma) \geq F(m, \sigma) F(n, \sigma)
$$

and $F(n, \sigma) \geq 1$; hence by Fekete's lemma on subadditive sequences,

$$
c(\sigma):=\lim _{n \rightarrow \infty} F(n, \sigma)^{1 / n} \in[1, \infty] \text { exists, }
$$

and $\forall n \geq 1, \quad F(n, \sigma) \leq c(\sigma)^{n}$.

Proof. First we will show (2) by constructing, from an $m$-permutation and an $n$ permutation which avoid $\tau$, an $(m+n)$-permutation which avoids $\tau$, injectively.

Without loss of generality, we may assume that $k$ precedes 1 in $\sigma$ (since, with $(\cdot)^{r}$ to denote the left-right reverse of a permutation, $\tau$ avoids $\sigma$ iff $\tau^{r}$ avoids $\sigma^{r}$, and hence for all $n, F(n, \sigma)=F\left(n, \sigma^{r}\right)$.) 
Let $\tau^{\prime} \in \mathcal{S}_{m}$ and $\tau^{\prime \prime} \in \mathcal{S}_{n}$, where each of $\tau^{\prime}$ and $\tau^{\prime \prime}$ avoids $\sigma$. Let $\tau^{\prime \prime \prime}$ be the result of adding $m$ to each symbol in the word for $\tau^{\prime \prime}$, so that $\tau^{\prime \prime \prime}$ is a word in which each of the symbols $m+1, \ldots, m+n$ appears exactly once.

Consider the concatenation $\tau$ of $\tau^{\prime}$ with $\tau^{\prime \prime \prime}$ as a permutation, $\tau \in \mathcal{S}_{m+n}$. Clearly, $\tau$ avoids $\sigma$, establishing (2).

[In detail, suppose to the contrary that $\tau$ contains $\sigma$, say at the $k$-tuple of positions given by $1 \leq x_{1}<x_{2}<\cdots<x_{k} \leq m+n$. Recall that $k$ precedes 1 in $\sigma$; say that $\sigma(a)=1$ and $\sigma(b)=k$ with $1 \leq b<a \leq k$, so that by (1), for $1 \leq i \leq k$, $\tau\left(x_{a}\right) \leq \tau\left(x_{i}\right) \leq \tau\left(x_{b}\right)$. If $x_{k} \leq m$ then $\tau^{\prime}$ contains $\sigma$, and if $x_{1}>m$ then $\tau^{\prime \prime}$ contains $\sigma$. If neither of these, then the $x_{1} \leq m$ so that $\tau\left(x_{1}\right) \leq m$, hence $\tau\left(x_{a}\right) \leq \tau\left(x_{1}\right) \leq m$ and therefore $x_{a} \leq m$; similarly $x_{k}>m$ so that $\tau\left(x_{k}\right)>m$, hence $\tau\left(x_{b}\right) \geq \tau\left(x_{k}\right)>m$ and therefore $x_{b}>m$, contradicting $b<a$.]

Recalling that $k$ precedes 1 in $\sigma$, the identity permutation in $\mathcal{S}_{n}$ avoids $\sigma$ and demonstrates that $F(n, \sigma) \geq 1$ for every $n \geq 1$. Fekete's lemma [4], see also [9], is that if $a_{1}, a_{2}, \ldots \in \mathbb{R}$ satisfy for all $m, n \geq 1, a_{m}+a_{n} \leq a_{m+n}$, then $\lim _{n \rightarrow \infty} a_{n} / n=$ $\inf _{n \geq 1} a_{n} / n \in[-\infty, \infty)$. Applying this with $a_{n}:=-\log F(n, \sigma)$ completes our proof.

There exist [10] examples with $\sigma, \sigma^{\prime} \in \mathcal{S}_{k}$, with $\sigma^{\prime}$ the identity permutation, and $F(n, \sigma)>F\left(n, \sigma^{\prime}\right)$, and Bóna [2], Theorem 4 shows that for all $n \geq 7, F(n, 1324)>$ $F(n, 1234)$. Nevertheless, it is possible that for every $k$, the largest exponential growth rate is the $(k-1)^{2}$ achieved by the identity permutation.

Conjecture 1. (\$100.00) For all $\sigma \in \mathcal{S}_{k}$ and $n \geq 1, F(n, \sigma) \leq(k-1)^{2 n}$.

\section{The problem of the shortest common superpattern.}

Define $G(n, k)$ to be the number of permutations $\tau \in \mathcal{S}_{n}$ which avoid at least one permutation in $\mathcal{S}_{k}$, i.e.

$$
G(n, k):=\left|\cup_{\sigma \in \mathcal{S}_{k}} \mathcal{A}(n, \sigma)\right|, \text { where } F(n, \sigma):=|\mathcal{A}(n, \sigma)| .
$$

Simion and Schmidt [8], p. 398, give a formula for $n !-G(n, 3)$, the number of $n$-permutations which contain all six patterns of length 3 . In considering $G(n, k)$, it is natural to consider the length $m(k)$ of the shortest permutation which contains every $\sigma \in \mathcal{S}_{k}$ as a subpattern, i.e. to consider

$$
m(k):=\min \{n: G(n, k)<n !\}=\min \left\{n: \cup_{\sigma \in \mathcal{S}_{k}} \mathcal{A}(n, \sigma) \neq \mathcal{S}_{n}\right\} .
$$

For a trivial lower bound on $m(k)$, since $\tau \in \mathcal{S}_{n}$ contains at most $\left(\begin{array}{l}n \\ k\end{array}\right)$ subpatterns, to contain every subpattern requires $\left(\begin{array}{l}n \\ k\end{array}\right) \geq k$ ! hence $\liminf _{k} m(k) / k^{2} \geq 1 / e^{2}$.

Theorem 2. There exists an n-permutation, with $n=k^{2}$, containing every $k$-permutation as a subpattern; i.e. $m(k) \leq k^{2}$. 
Proof. Consider the lexicographic order on $[k]^{2}$ as a one-to-one map specifying the ranks of the ordered pairs, i.e. let $r:[k]^{2} \rightarrow\left[k^{2}\right]$, with $(i, j) \mapsto(i-1) k+j$. Also consider the transposed lexicographic order $t:[k]^{2} \rightarrow\left[k^{2}\right]$ given by $t(i, j):=r(j, i)$. Consider the permutation $\tau \in \mathcal{S}_{k^{2}}$ given by $\tau=r \circ t^{-1}$; for example, with $k=3$, this is $\tau=147258369$. Then, clearly, $\tau$ contains every $\sigma \in \mathcal{S}_{k}$ as a subpattern. In detail, with the positions $x_{1}:=t(\sigma(1), 1), \ldots, x_{k}:=t(\sigma(k), k)$ we have $x_{1}<\cdots<x_{k}$ and for $m=1$ to $k, \tau\left(x_{m}\right)=\left(r \circ t^{-1}\right)(t(\sigma(m), m))=r(\sigma(m), m)$ so that $\tau\left(x_{a}\right)<\tau\left(x_{b}\right)$ iff $\sigma(a)<\sigma(b)$.

Conjecture 2. As $k \rightarrow \infty, m(k) \sim(k / e)^{2}$.

In contrast, from the known behavior of the length $L_{n}$ of the longest increasing subsequence, $L_{n} \sim 2 \sqrt{n}$ with high probability, one cannot hope to use random permutations to show that $\lim \inf m(k) / k^{2} \leq(1 / e)^{2}$. The probability that a random $n$-permutation does not contain every $\sigma \in \mathcal{S}_{k}$ as a subpattern is $G(n, k) / n$ !. Define the threshold $t(k)$ by $t(k)=\min \{n: G(n, k) / n ! \leq 1 / 2\}$, so that trivially $m(k) \leq t(k)$, and hence $\lim \inf t(k) / k^{2} \geq 1 / 4$.

Conjecture 3. (Noga Alon) The threshold length $t(k)$, for a random permutation to contain all $k$-permutations with substantial probability, has $t(k) \sim(k / 2)^{2}$.

\section{REFERENCES}

[1] Alon, N., and Friedgut, E. (1999) On the number of permutations avoiding a given pattern. J. Combinatorial Theory, Ser. A, to appear

[2] Bóna, M. (1997) Exact and asymptotic enumeration of permutations with subsequence conditions. Ph.D. Thesis, M.I.T.

[3] Bóna, M. (1999) The solution of a conjecture of Stanley and Wilf for all layered patterns. J. Combinatorial Theory, Ser. A 85, 96-104.

[4] Fekete, M. (1923) Über die Verteilung der Wurzeln bei gewissen algebraischen Gleichungen mit ganzzähligen Koeffizienten. Math. Z. 17, 228-249.

[5] Klazar, M. (1992) A general upper bound in extremal theory of sequences. Comment. Math. Univ. Carolin. 33, 737-746.

[6] Knuth, D. E. (1968) The art of computer programming. Addison-Wesley, Reading, MA.

[7] Regev, A. (1981) Asymptotic values for degrees associated with strips of Young diagrams. Adv. Math. 41, 115-136.

[8] Simion, R., and Schmidt, F. W. (1985) Restricted permutations. European J. of Combinatorics 6, 383-406.

[9] Steele, J. M. (1997) Probability theory and combinatorial optimization. CBMS-NSF regional conference series in applied mathematics 69. SIAM, Philidelphia, PA.

[10] West, J. (1990) Permutations with forbidden subsequences; and stack sortable permutations. Ph.D. Thesis, MIT. 